\title{
Fitting superellipses to incomplete contours
}

\author{
M. Osian \\ T. Tuytelaars \\ K. U. Leuven, ESAT/PSI \\ K. U. Leuven, ESAT/PSI \\ mosian@esat.kuleuven.ac.be \\ tuytelaa@esat.kuleuven.ac.be \\ L. Van Gool \\ K.U. Leuven \& ETH Zurich \\ vangool@esat.kuleuven.ac.be
}

\begin{abstract}
Affine invariant regions have proved a powerful feature for object recognition and categorization. These features heavily rely on object textures rather than shapes, however. Typically, their shapes have been fixed to ellipses or parallelograms. The paper proposes a novel affine invariant region type, that is built up from a combination of fitted superellipses. These novel features have the advantage of offering a much wider range of shapes through the addition of a very limited number of shape parameters, with the traditional ellipses and parallelograms as subsets. The paper offers a solution for the robust fitting of superellipses to partial contours, which is a crucial step towards the implementation of the novel features.
\end{abstract}

\section{Introduction}

Quite recently, affine invariant regions have made a rather impressive entrance into computer vision (e.g. $[1,7,9,13$, 15]). Soon, these features have shown to have great potential for some of the long-standing problems in computer vision such as viewpoint-independent object recognition (e.g. [12]), wide baseline matching (e.g. [14]), object categorization (e.g. [2,3]) and texture classification (e.g. [6]).

Affine invariant regions in a way ran contrary to what had been the dominant credo in the recognition literature up to that point, namely that shapes, parts, and contours were the crucial features, not texture. Yet, none of the shape related strategies had ever been able to reach the same level of performance. Intuitively, it is difficult to accept that shape shouldn't play a bigger role. Also, strategies based on affine invariant regions have not been demonstrated to recognize untextured objects and therefore offer only a partial solution. We propose a generalization of affine invariant regions. In contrast to those proposed in literature, these regions do adapt their shapes to that of the local object contours. They are based on the fitting of affinely deformed superellipses to contour segments. By combining several, partial superellipses a wide variety of region shapes can be generated with the addition of only few parameters.
The paper is structured as follows: Section 2 introduces the family of shapes called "affine superellipses". Section 3 presents our approach to fitting affine superellipses to partial contours. Section 4 shows some preliminary results that we obtained. Conclusions are drawn in Section 5.

\section{Affine superellipses}

Ellipses and parallelograms are ideal shapes to build affine invariant regions from, because both families of shapes are closed under affine transformations. On the other hand, they are quite restrictive in terms of the possible shapes. There is a family of curves, however, that takes one additional parameter, and generates a much wider class of shapes. These are the so-called 'superellipses'. Superellipses were introduced in 1818 by the French mathematician Gabriel Lamé. Their Cartesian equation is [16]:

$$
\left|\frac{x}{a}\right|^{r}+\left|\frac{y}{b}\right|^{r}=1
$$

To avoid the modulus, the above formula can be written as a function of $x^{2}, y^{2}$ and an exponent $\varepsilon$ [5]. We first consider the particular case when the scaling coefficients $a$ and $b$ are both 1 . We call this initial family of shapes "supercircle" of unit radius (see Fig. 1):

$$
\left(x^{2}\right)^{\varepsilon}+\left(y^{2}\right)^{\varepsilon}=1
$$

The addition of the single parameter $\varepsilon$ yields an interesting variety of shapes. Next we generalize this shape family to one that is closed under affine transformations, more precisely shapes that can be reduced to a supercircle via an

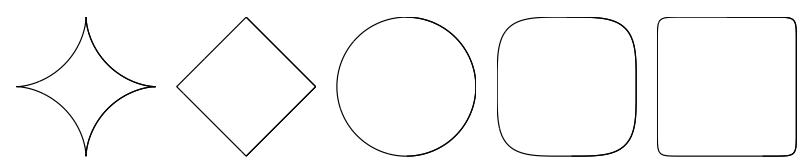

Figure 1: "Supercircles" for different values of $\varepsilon$ : $0.3,0.5,1,2,8$ 


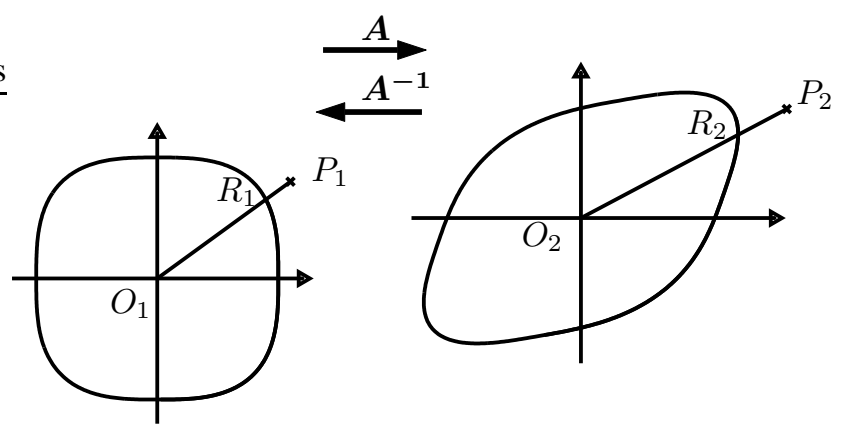

Figure 2: Result of applying an affine transformation $A$ to a supercircle.

affine transformation. The rationale is that as in the case of existing affine invariant regions, we want to find corresponding regions under variable viewpoints. These changes can be represented well by affine transformations. Hence, points $\overrightarrow{x_{e}}$ on these shapes are found as:

$$
\overrightarrow{x_{e}}=A \overrightarrow{x_{c}}
$$

where $\overrightarrow{x_{c}}$ verifies the supercircle equation (1) and $A$ is shorthand for the $3 \times 3$ affine transformation matrix. This family of shapes is wider than that of the original superellipses. Not only does it allow for rigid motions of the superellipses, but it also includes skewed versions, as exemplified in fig. 2 . Applying affine transformations to superellipses rather than supercircles leads to exactly the same family, but with an over-parameterized representation.

We refer to the family as affine superellipses or ASEs for short. The parameter $\varepsilon$ provides a viewpoint independent shape parameter. If we can compose curves with a small set of well fitting ASEs, the corresponding $\varepsilon s$ and the ASEs' configuration provide compact and viewpoint independent shape information. Fitting ASEs is the subject of the next section.

\section{ASE fitting}

The problem of fitting superellipses is not entirely new. Rosin [11] has compared several objective functions to be minimized. These functions represent summed distances between the data points and selected points on the model curve. It proved difficult to choose one that would perform best in all cases. An important limitation was that contours were supposed to be closed. Fitting of an initial bounding box allowed him to immediately get rid of the translation and rotation components in the optimization. In a subsequent paper Zhang and Rosin [18] generalized the optimization to partial contours. They also mapped shapes back to the circle, as a normalization step prior to the evaluation of the objective function. The latter consisted of a sum of algebraic distances between the normalized contour and the circle, taking the local contour gradient and curvature into account.

In our work, we have to deal with partial contours. We also add the skew parameter in order to deal with the full set of ASEs. Moreover, using the algebraic distance depends exponentially on $\varepsilon$. For example, considering the point $(x, y)$ on the unit radius supercircle, the point $(x+d, y)$ yields the error $(x+d)^{2 \varepsilon}-x^{2 \varepsilon}$. This means that rectangular shapes $(\varepsilon \gg 1)$ are more sensitive to outliers. Therefore, we used the Euclidean distance between contour points and the intersection of the ASE with the join through the points and the ASE's center. Notice that this procedure does not normalize the ASE to a supercircle and, hence, the fitting procedure is not strictly affine invariant. We have found that prior normalization yields fitting results that are less robust, however. Some examples illustrating this are shown in fig. 3. We still need to study the precise causes in more depth. It should be noted that the Zhang and Rosin approach is also not affine invariant, even if they normalize, as they evenly sample the image contour before normalizing. Affine invariance wasn't part of their goals. This sampling problem is shared by the PCA-based methods proposed by Pilu et al. [10], which deal with larger sets of deformations than affine, but only for closed contours.

With the notations from fig. 2, we minimize the sum of all squared Euclidean distances $P_{2} R_{2}$, where $P_{2}$ represents a data point and $R_{2}$ is the intersection between the ASE and the line passing through $\mathrm{P}_{2}$ and $\mathrm{O}_{2}$ - the ASE's center:

$$
D=\sum_{P_{2} \in \text { data }}\left|\overrightarrow{P_{2}}-\vec{R}_{2}\right|^{2}
$$

The location of $R_{2}$ is computed as follows:

$$
\overrightarrow{R_{2}}=A \overrightarrow{R_{1}}
$$

where $A$ is an affine matrix expressing the translation $T$, rotation $R$, scale $S$ and skew $K$ of the ASE:

$$
A=T R S K
$$

$$
\begin{aligned}
T=\left[\begin{array}{ccc}
1 & 0 & t_{x} \\
0 & 1 & t_{y} \\
0 & 0 & 1
\end{array}\right] ; \quad R=\left[\begin{array}{ccc}
\cos \theta & \sin \theta & 0 \\
-\sin \theta & \cos \theta & 0 \\
0 & 0 & 1
\end{array}\right] \\
S=\left[\begin{array}{ccc}
s_{x} & 0 & 0 \\
0 & s_{y} & 0 \\
0 & 0 & 1
\end{array}\right] ; \quad K=\left[\begin{array}{ccc}
1 & k & 0 \\
0 & 1 & 0 \\
0 & 0 & 1
\end{array}\right]
\end{aligned}
$$

Switching to polar coordinates, $R_{1}$ becomes:

$$
\overrightarrow{R_{1}}(\rho, \theta):\left\{\begin{array}{l}
x_{R_{1}}=\rho_{R_{1}} \cos \theta_{R_{1}} \\
y_{R_{1}}=\rho_{R_{1}} \sin \theta_{R_{1}}
\end{array}\right.
$$



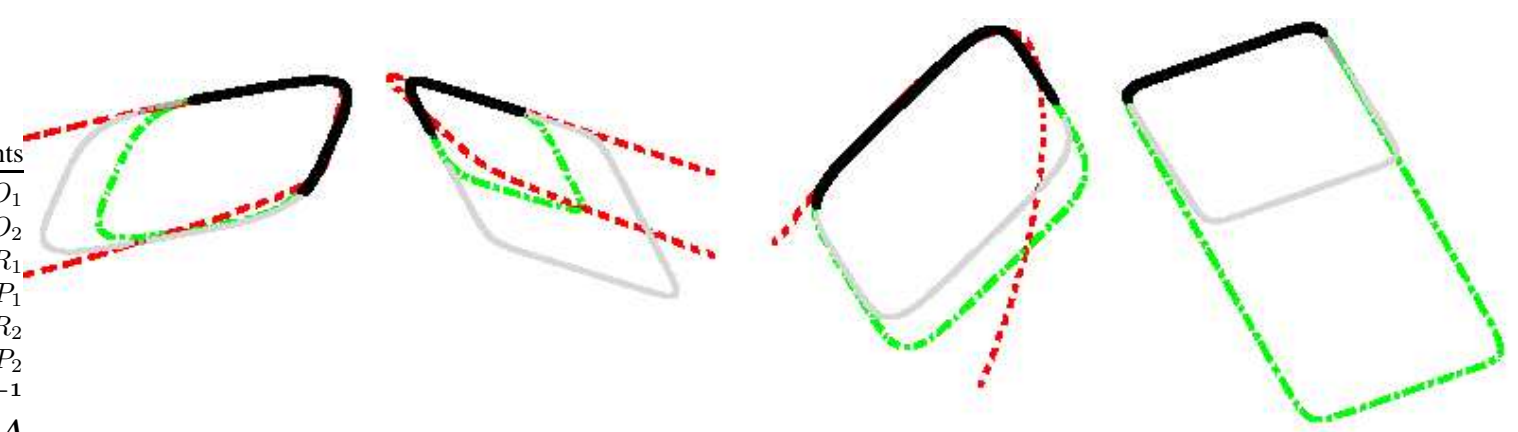

Figure 3: Fitting superellipses to partial data. The red dashed lines represent the results of fitting using normalized distance, dotted green lines represent fitting using image distance. The original contours are gray. The segments used for fitting are drawn in black. In the last case the normalized version failed to converge.

$\overrightarrow{R_{1}}$ verifies the supercircle equation (1):

$$
\begin{aligned}
& \left(\rho_{R_{1}}^{2}\right)^{\varepsilon}\left(\left(\cos ^{2} \theta_{R_{1}}\right)^{\varepsilon}+\left(\sin ^{2} \theta_{R_{1}}\right)^{\varepsilon}\right)=1 \\
& \Leftrightarrow \rho_{R_{1}}=\left(\left(\cos ^{2} \theta_{R_{1}}\right)^{\varepsilon}+\left(\sin ^{2} \theta_{R_{1}}\right)^{\varepsilon}\right)^{\frac{-1}{2 \varepsilon}}
\end{aligned}
$$

$P_{1}$ and $R_{1}$ are colinear, so by replacing

$$
\left\{\begin{aligned}
\cos \theta_{R_{1}} & =\frac{x_{P_{1}}}{\rho_{P_{1}}} \\
\sin \theta_{R_{1}} & =\frac{y_{P_{1}}}{\rho_{P_{1}}}
\end{aligned}\right.
$$

in equations (6) and (8), $\overrightarrow{R_{1}}$ can be written as a function of $\vec{P}_{1}$ and $\varepsilon$ :

$\overrightarrow{R_{1}}=f\left(\vec{P}_{1}, \varepsilon\right) \Leftrightarrow\left\{\begin{array}{l}x_{R_{1}}=x_{P 1}\left(\left(x_{P_{1}}^{2}\right)^{\varepsilon}+\left(y_{P_{1}}^{2}\right)^{\varepsilon}\right)^{\frac{-1}{2 \varepsilon}} \\ y_{R_{1}}=y_{P 1}\left(\left(x_{P_{1}}^{2}\right)^{\varepsilon}+\left(y_{P_{1}}^{2}\right)^{\varepsilon}\right)^{\frac{-1}{2 \varepsilon}}\end{array}\right.$

Also, $\overrightarrow{P_{1}}=A^{-1} \vec{P}_{2}$, so the expression of $\overrightarrow{R_{2}}$ is:

$$
\overrightarrow{R_{2}}=A f\left(A^{-1} \overrightarrow{P_{2}}, \varepsilon\right)
$$

Finally, the objective function has the following expression:

$$
D=\sum_{P_{2} \in \text { data }}\left|\overrightarrow{P_{2}}-A f\left(A^{-1} \vec{P}_{2}, \varepsilon\right)\right|^{2}
$$

This being a nonlinear least-squares minimization problem, we applied the Levenberg-Marquardt algorithm [17], a very effective and popular method for this category. The Levenberg-Marquardt algorithm requires an initial estimate of the objective function's parameters, then proceeds iteratively towards the minimum. At each iteration it needs to evaluate the residual error and the function's Jacobian matrix. The Jacobian has a quite complicated form, but the computations are straightforward so we will omit them here.
The $\varepsilon$ parameter is initialized to 2 and the skew to 0 , while the other coefficients of the matrix $A$ are initialized by an ellipse fitting algorithm [4]. The condition set for stopping the Levenberg-Marquardt algorithm is that for all of the 7 parameters the difference between successive iterations is less than $10^{-8}$. On average less than 20 iterations are required for convergence.

\subsection{Contour extraction}

An important issue is how the contours are extracted from natural images. Good contours are rather difficult to find, due to textures, occlusions, shadows, etc. For testing our algorithm we used a method introduced by Tuytelaars et al. [15]. Starting from a local extremum in the intensity $K(x, y)$, rays are shot under different angles. The intensity pattern along each ray emanating from the extremum is studied by evaluating the function

$$
f_{K}(t)=\frac{\left|K(t)-K_{0}\right|}{\max \left(d, \frac{1}{t} \int_{0}^{t}\left|K(\tau)-K_{0}\right| d \tau\right)}
$$

with $t$ being the Euclidean arc length along the ray, $K(t)$ the intensity at position $t, K_{0}$ the intensity extremum and $d$ a small number added to prevent division by zero. The point at which this function reaches an extremum is invariant under the affine geometric and photometric transformations (given the ray). All points corresponding to an extremum of $f_{K}$ along the rays are linked to form a closed contour. These are the contours from which we select affine invariant segments and then fit ASEs against.

\subsection{Selection of invariant contour segments}

When fitting to partial contours, there is a further issue that corresponding segments should be selected independent of viewpoint. This can be achieved by using simple, affine 


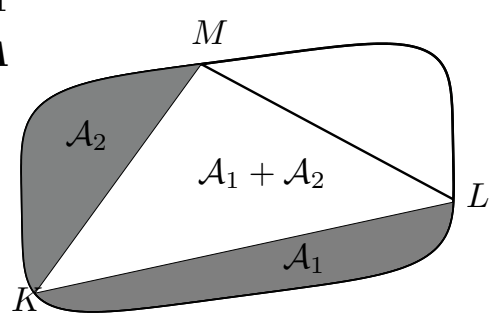

Figure 4: Automatic selection of a partial contour: Starting from $K$, the chords $K L$ and $K M$ are drawn such that the area of the $K L M$ triangle is equal to $\mathcal{A}_{1}+\mathcal{A}_{2}$. The partial contour excludes the $L M$ arc.

invariant criteria. One is illustrated in fig. 4. Starting from a point $K$, one can select a segment such that the chords from the point to each of the endpoints enclose the same area between them and the contour, i.e. $\mathcal{A}_{1}=\mathcal{A}_{2}$ in fig. 4. There typically is an infinite number of such segments still. Demanding that the white triangle $\triangle K L M$ in the figure has the two areas summed $\left(\mathcal{A}_{1}+\mathcal{A}_{2}=2 \mathcal{A}_{1}=2 \mathcal{A}_{2}\right)$ reduces the number of such segments to a finite set of possibilities. These segments M - K - L are the ones we have fitted the ASEs to.

When fitting we also look at the error. Local minima are of particular importance, as they suggest segments out of which the complete contour can be composed in a highly compact way. This point will be illustrated in the next section, where we show ASE-fits to contours extracted from real images.

\section{Experimental results}

\subsection{Synthetic data}

For testing the accuracy of the fitting module we generated noisy superellipses with different rotation, scaling, skew and epsilon coefficients. We fixed the horizontal scaling factor to 100, and modified the other parameters as follows: vertical scaling from 50 to 150 in 6 steps, rotation angle from 0 to $\pi / 2$ in 30 steps, skew factor from -50 to 50 in 6 steps. For each combination we modified the value of $\varepsilon$ from 1 to 30 in increments of 1 and verified the absolute error of the recovered $\varepsilon$ for different noise levels. The noise was generated from a uniform distribution, having a spread of $0,1,2$ and 4 pixels. Fitting was done using only half of the full contour. The coordinates of the generated points were rounded to integer coordinates. The plot of the standard deviation of the estimated $\varepsilon$ from the true value is shown in fig. 5. As it can be seen, rectangular shapes $(\varepsilon \gg 1)$ are the worst affected by noise and rounding. This

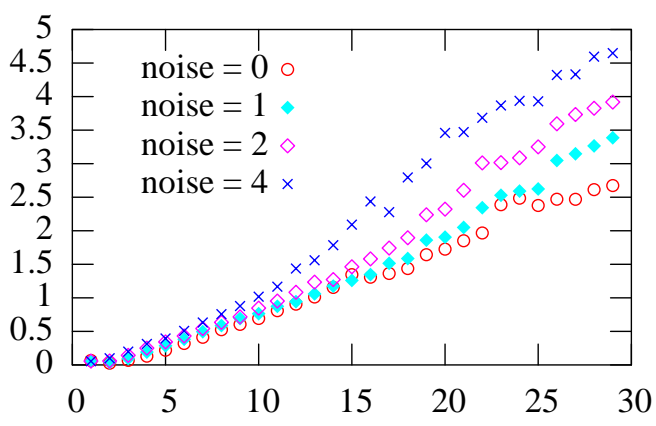

Figure 5: Plot of the error of $\varepsilon$ : The horizontal axis represents the ground truth $\varepsilon$. The vertical axis shows the standard deviation of the recovered $\varepsilon$.

result is not surprising, since sharp corners become less clear as the noise increases.

In the absence of noise and without rounding the coordinates to integers, the fitting procedure can recover the original $\varepsilon$ up to the fifth decimal.

\subsection{Natural image contours}

Fig. 6 shows the back windows of a car, viewed from two directions. We show two types of ASEs that yield local minima for the fitting error. As can be seen, these correspond quite well, both between the mirror symmetric window pairs within each image, but also between the images. The overall shape can be represented efficiently as a combination of the two ASE types, as can be seen in the right column. The epsilon values are added in the figure and can be seen to be quite clustered. The difference in the values of $\varepsilon$ is caused by systematic errors during the contour extraction. Similar examples can be seen in fig. 7 and 8. Fig. 7 and 9 show ASEs fitted to the headlights of different cars. Again, as few as 2 ASEs manage to form a good approximation of these shapes. As one can see, in contrast to e.g. wheels, which always are elliptical with $\varepsilon=1$, head-lights are parts with a much wider variation in their shapes.

As can also be seen from these examples, the pairs of head-lights are approximated by ASEs with similar epsilon values. Other examples can be seen in fig. 10 and 11 .

\section{Conclusions and future work}

Currently, we are working on affine invariant descriptions of such ASE configurations, both in terms of their overall shape, as the texture content within their approximated contour. For the latter, already extensive sets of measures exist. Moment invariants would be one option [8]. As to the shape features, the ratio of areas of the different ASEs in the 
final configuration would be one simple, additional example. Other features should describe their relative positions, skews, and orientations. These can be quantified by normalizing one of the ASEs to a supercircle, and expressing these parameters with respect to the reference frame thus created.

We have described quite preliminary work. Nevertheless the results seem to corroborate the viability of the ASE approach. In its full-fledged form it will not only include several of the affine invariant region types already in use, but will also provide a link between the texture based methods that these basically are, and shape-based approaches. Indeed, it stands to reason that a truly generic recognition system will have to draw on both.

\section{Acknowledgements}

The authors gratefully ackowledge support from EC Cognitive Systems project CogViSys and the fund for Scientific Research Flanders.

\section{References}

[1] A. Baumberg, "Reliable feature matching across widely separated views", IEEE Computer Vision and Pattern Recognition, pp. 774-781, 2000.

[2] M.C. Burl, M. Weber, T.K. Leung and P. Perona "Recognition of Visual Object Classes", Chapter to appear in: From Segmentation to Interpretation and Back: Mathematical Methods in Computer Vision, Springer Verlag.

[3] R. Fergus, P. Perona, A. Zisserman, "Object Class Recognition by Unsupervised Scale-Invariant Learning", Proc. of the IEEE Conf on Computer Vision and Pattern Recognition, 2003.

[4] A. Fitzgibbon, M. Pilu, R.B. Fisher, "Direct least squares fitting of ellipses", IEEE Transactions on Pattern Analysis and Machine Intelligence, Vol 21, Issue 5, pp. 476-480, May 1999.

[5] M. Gardiner, "The superellipse: a curve that lies between the ellipse and the rectangle", Scientific American 21, pp. 222-234, 1965.

[6] S. Lazebnik, C. Schmid, J. Ponce, "Affine-Invariant Local Descriptors and Neighborhood Statistics for Texture Recognition", Proceedings of the IEEE International Conference on Computer Vision, Nice, France, October 2003, pp. 649-655.

[7] K. Mikolajczyk, C. Schmid, "An affine invariant interest point detector", European Conference on Computer Vision, Vol. 1, pp. 128-142, 2002.
[8] F. Mindru, T. Moons, L. Van Gool, "Recognizing color patterns irrespective of viewpoint and illumination", Proceedings Conference on Computer Vision and Pattern Recognition - CVPR, IEEE, pp. 368-373, June 1999.

[9] S. Obdzalek, J.Matas, "Object recognition using Local Affine Frames on Distinguished Regions", British Machine Vision Conference, pp. 414-431, 2002.

[10] M. Pilu, A.W. Fitzgibbon, and R.B. Fisher, "Training PDM on models: The case of deformable superellipses", In Proceedings of the British Machine Vision Conference, Edinburgh, pp. 373-382, September 1996.

[11] P. Rosin, "Fitting Superellipses", IEEE Transactions on Pattern Analysis and Machine Intelligence, Vol 22, No. 7, July 2000.

[12] F. Rothganger, S. Lazebnik, C. Schmid, J. Ponce, "3D Object Modeling and Recognition Using AffineInvariant Patches and Multi-View Spatial Constraints", Proceedings of the IEEE Conference on Computer Vision and Pattern Recognition, Madison, WI, June 2003, Vol. II, pp. 272-277.

[13] C. Schmid, R. Mohr, "Local Grayvalue Invariants for Image Retrieval", IEEE Transactions on Pattern Analysis and Machine Intelligence, 19(5), pp. 530-535, 1997.

[14] D. Tell, S. Carlsson, "Wide baseline point matching using affine invariants", ECCV, Vol. 1, pp. 814-828, 2000 .

[15] T. Tuytelaars, L.Van Gool, "Wide baseline stereo matching based on local affinely invariant regions", Proceedings British Machine Vision Conference, Sept.2000, pp. 412-422.

[16] Eric W. Weisstein. "Superellipse." From MathWorld A Wolfram Web Resource. http://mathworld.wolfram.com/Superellipse.html

[17] Eric W. Weisstein. "Levenberg-Marquardt Method." From MathWorld - A Wolfram Web Resource. http://mathworld.wolfram.com/LevenbergMarquardt Method.html

[18] X. Zhang, P. Rosin "Superellipse fitting to partial data", Pattern Recognition, No. 36, pp. 743-752, 2003. 

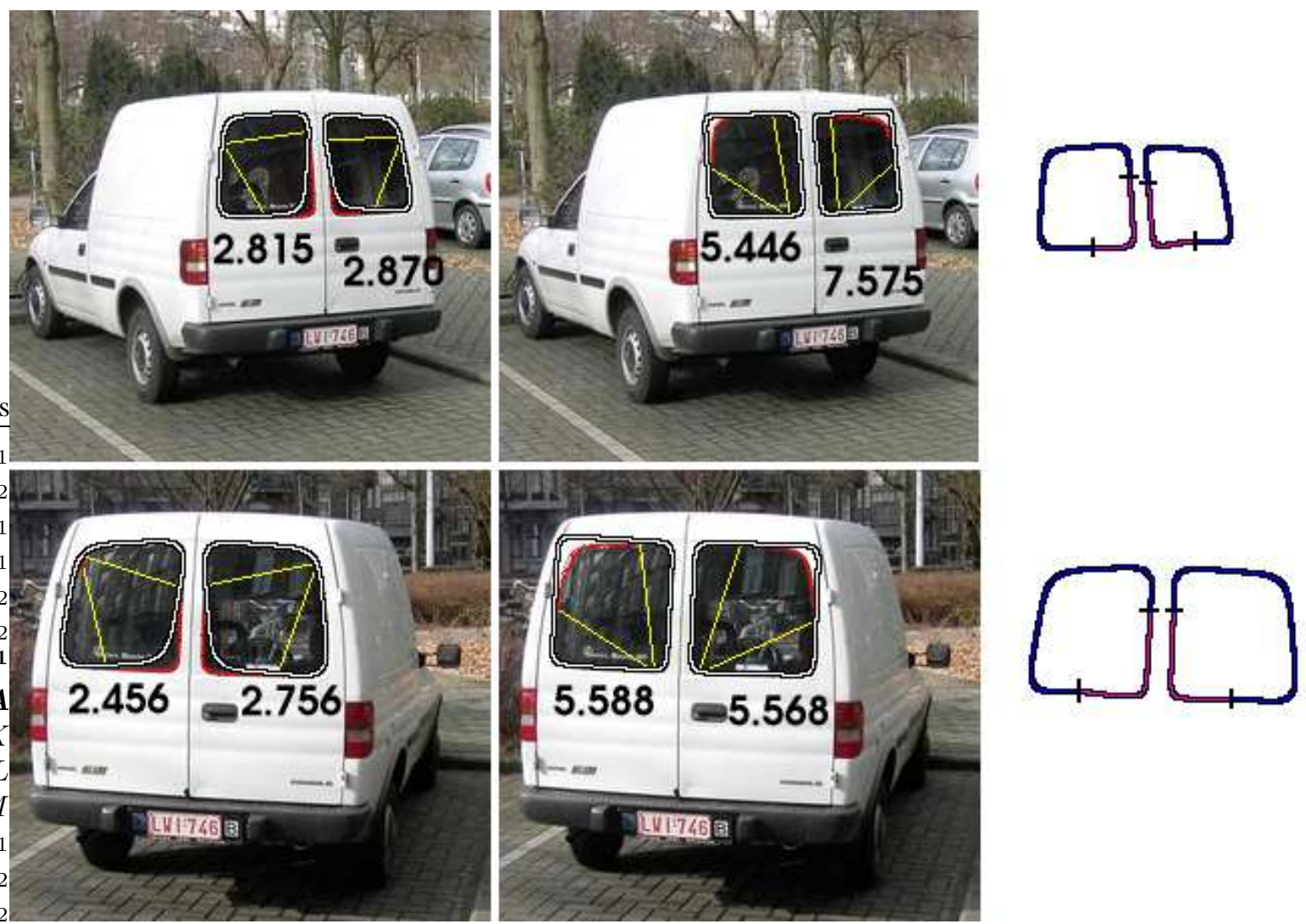

Figure 6: A combination of two ASEs can approximate the rear window of a car.
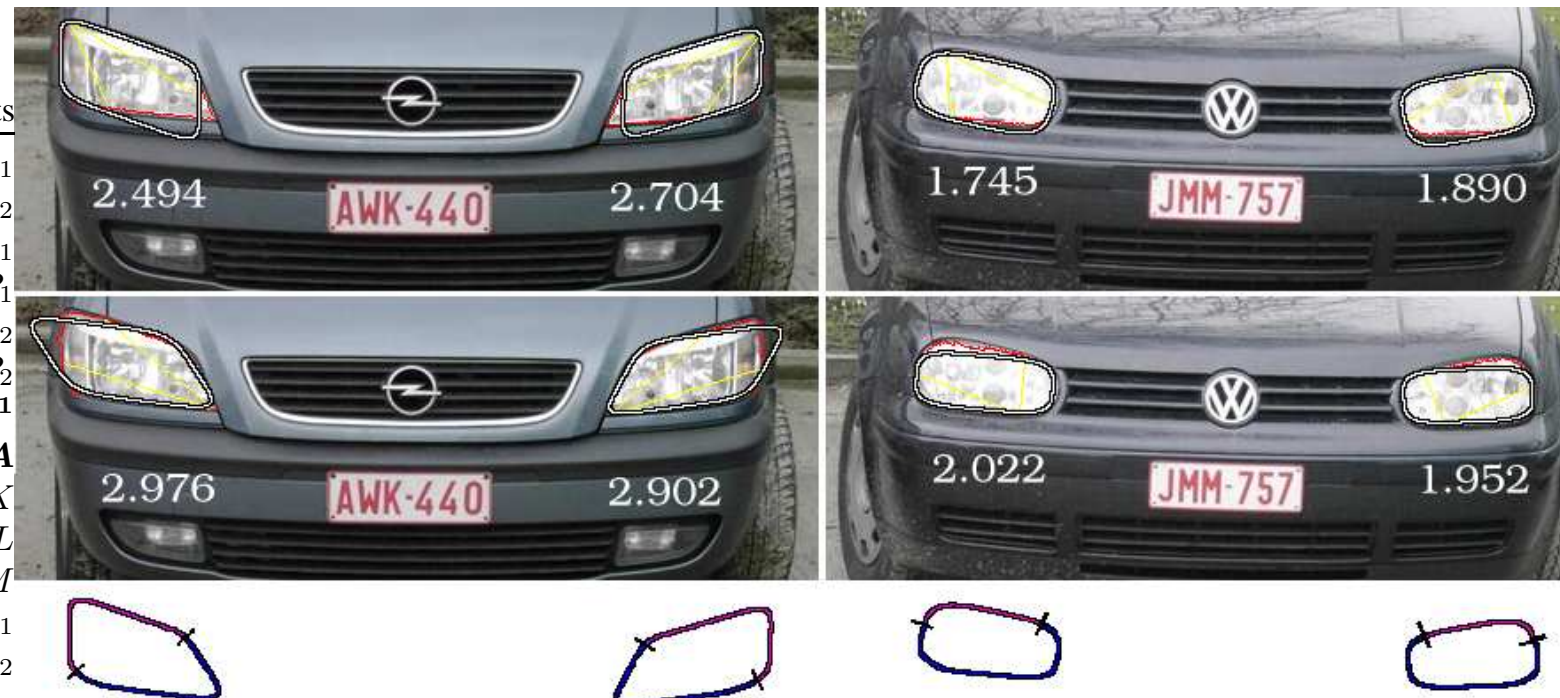

Figure 7: Car headlights represented with ASE combinations. 


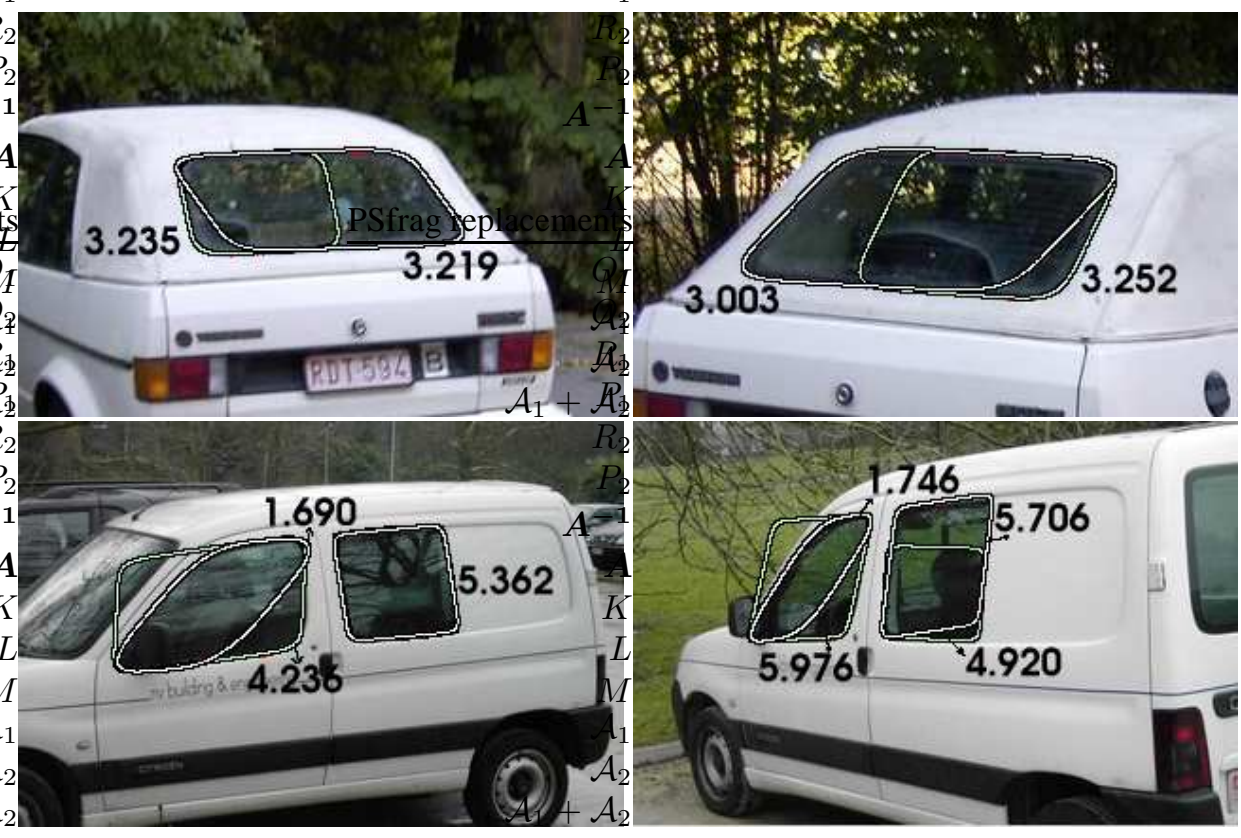

Figure 8: Viewpoint invariance. Note that the bottom-right image is affected by projective errors, thus the rectangular window must be approximated by two ASEs.
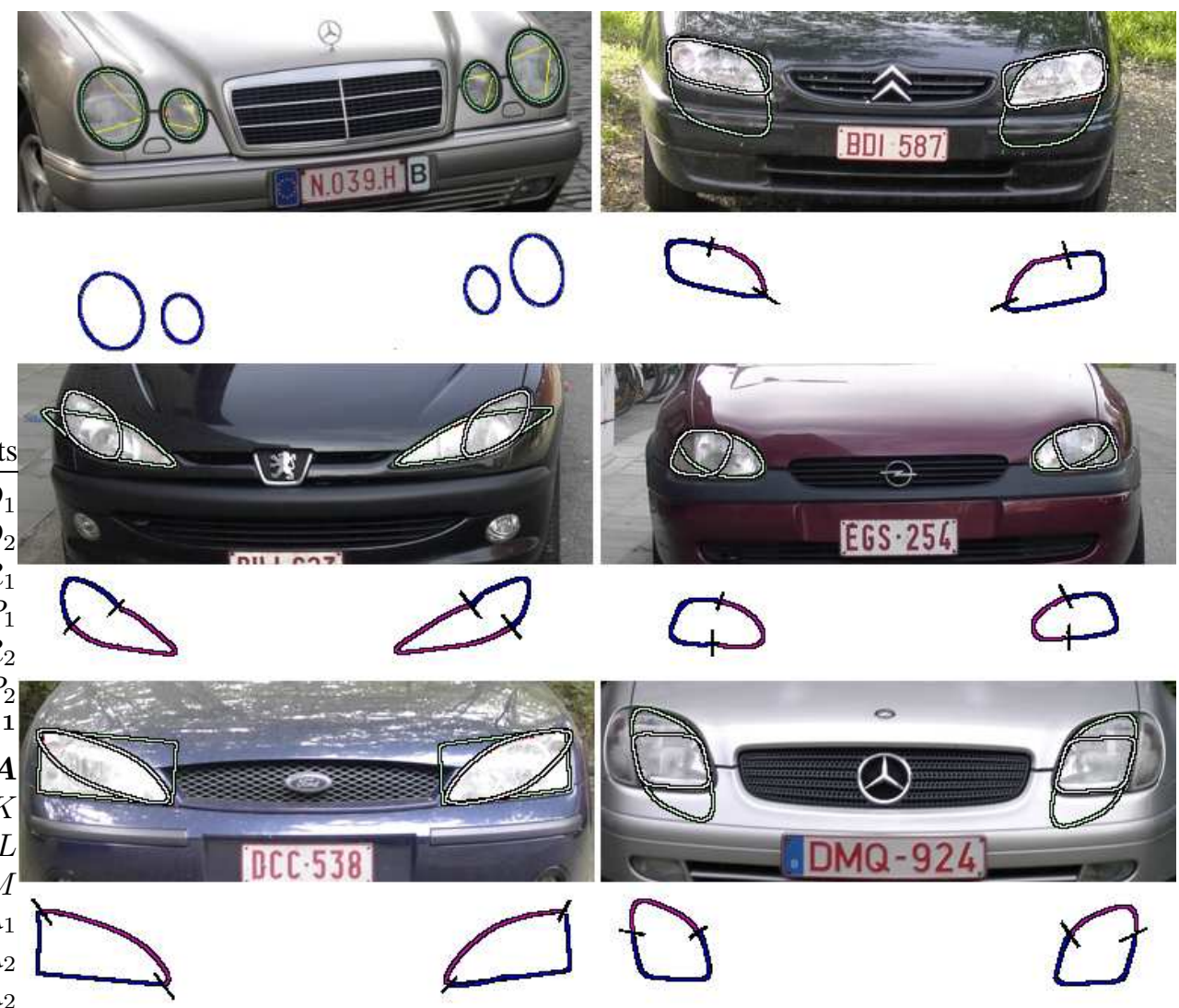

Figure 9: Other ASE combinations fitted to car headlights. 

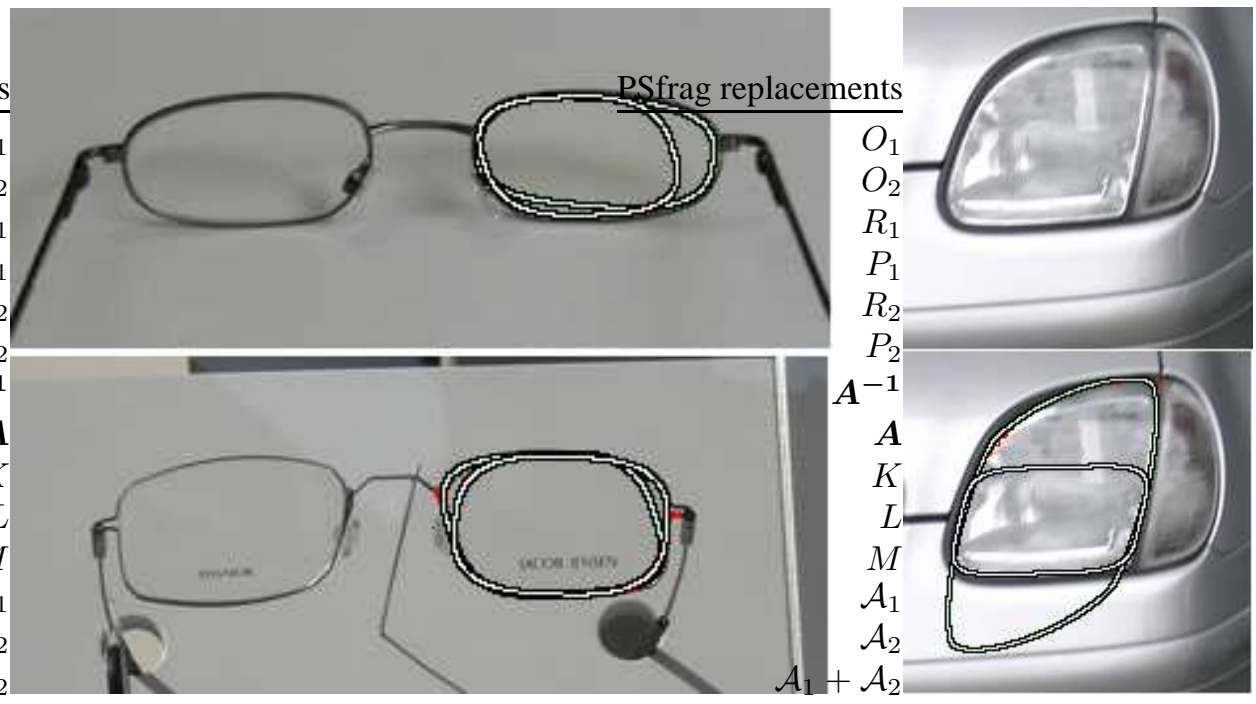

Figure 10: Left: two pairs of glasses. Right: Only a part of the headlight has been detected by the contour extraction module.
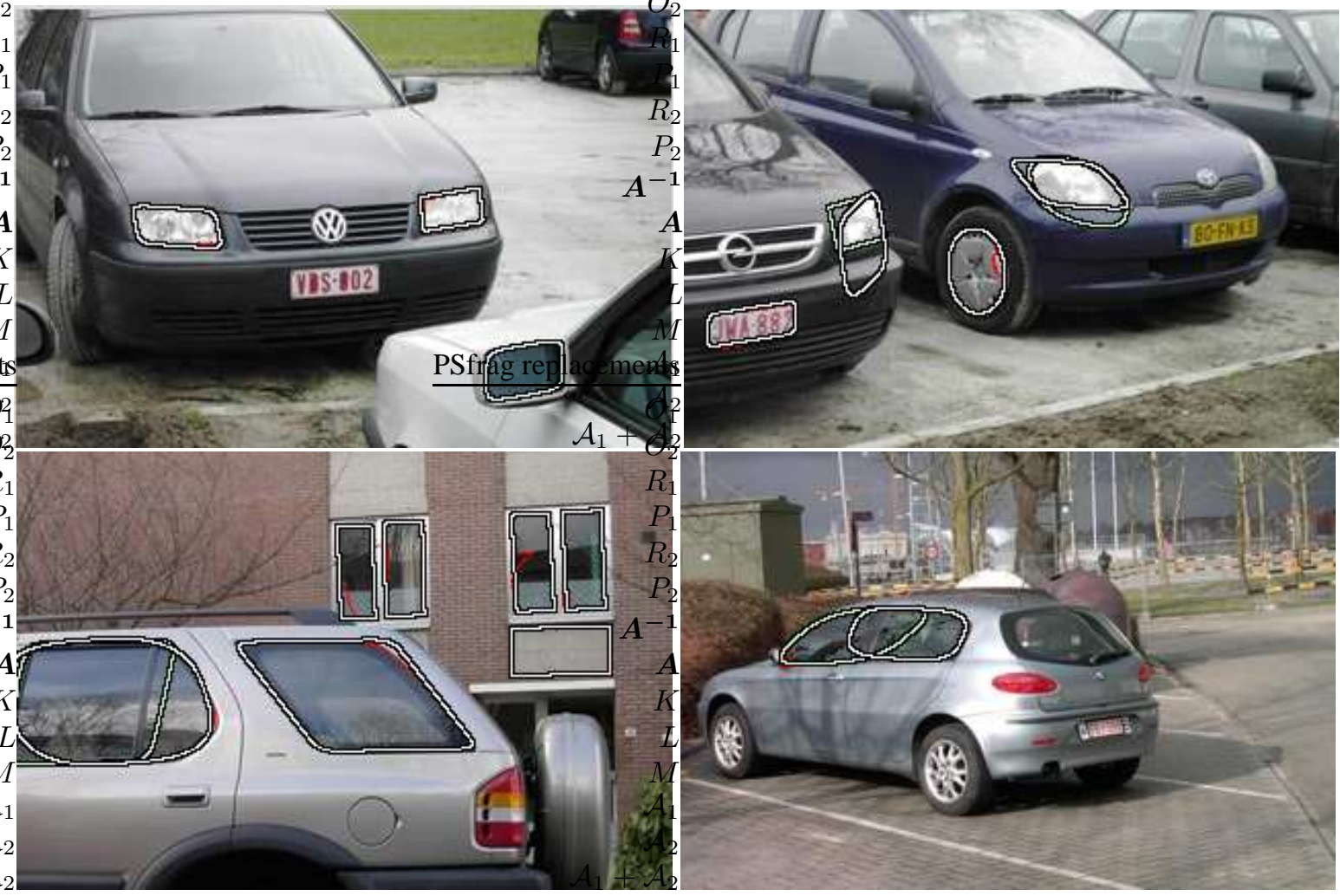

Figure 11: Other examples of ASEs. 